\title{
MEK5-ERK5 pathway associates with poor survival of breast cancer patients after systemic treatments
}

\author{
Mariska Miranda ${ }^{1}$, Esdy Rozali ${ }^{1}$, Kum Kum Khanna ${ }^{1}$ and Fares Al-Ejeh ${ }^{1}$ \\ ${ }^{1}$ QIMR Berghofer Medical Research Institute, Herston QLD, Australia. \\ Correspondence to: Fares Al-Ejeh, email: Fares.Al-Ejeh@qimrberghofer.edu.au \\ Keywords: ERK5/MAPK7, MEK5/MAP2K5, triple negative breast cancer, TNBC, chemo-resistance \\ Received: February 02, $2015 \quad$ Accepted: February 18, $2015 \quad$ Published: February 20, 2015
}

This is an open-access article distributed under the terms of the Creative Commons Attribution License, which permits unrestricted use, distribution, and reproduction in any medium, provided the original author and source are credited.

\section{ABSTRACT}

The MEK5-ERK5 pathway is a mammalian mitogen-activated protein (MAP) kinase cascade that is not well studied compared to other MAP kinase cascades. Two independent studies by Al-Ejeh et al. and Ortiz-Ruiz et al. published in Oncotarget last year concluded that ERK5 is an attractive target in triple negative breast cancer. In this perspective, we briefly describe the findings of these studies and propose the use of pharmacological inhibition of ERK5 in combination with chemotherapy against triple negative breast cancer because MEK5-ERK5 overexpression associates with poor survival of patients treated with chemotherapy.

ERK5 (MAPK7) consists of the N-terminal kinase domain that is highly homologous to the more studied MAP kinase ERK1/2. However, ERK5 - also called the big mitogen activated protein kinase 1 (BMK1) - contains a unique large $\mathrm{C}$-terminal domain which does not exist in other MAP kinases $[1,2]$. Two articles published in Oncotarget in 2014 characterized ERK5 as a therapeutic target against triple negative breast cancer [3, 4]. Using the Kinex ${ }^{\mathrm{TM}}$ antibody arrays to profile primary breast tumors, we found that a subset of triple negative breast cancer (TNBC) overexpress ERK5 and its upstream activator MEK5. TNBC cell lines were sensitive to the specific ERK5 inhibitor (XMD8-92) and the MEK5/ERK5 inhibitor (BIX02188). We found that the ERK5 inhibition alone and more so with anthracycline/taxane-based chemotherapy had an anti-tumour response against TNBC tumors in vivo. This anti-tumor response was associated with increased DNA damage and apoptotic cell death [3]. Ortiz-Ruiz et al. [4] reported the overexpression of ERK5 in TNBC and that its inhibition with the CDK/ERK5 inhibitor TG02 [5] induced apoptotic cell death in vitro and with an anti-tumor effect in vivo. The authors also reported that ERK5 inhibition potentiated chemotherapy in vitro. In both studies, members of the Bcl-2 family were modulated by ERK5 inhibition explaining the anti-tumor effect of ERK5 inhibitors [3, 4].

Ortiz-Ruiz et al. [4] interrogated published gene expression data from breast cancer using the Kaplan-
Meier Online Tool (KM Plotter) [6] and found that ERK5 mRNA overexpression associated with poor relapsefree survival in node-positive basal-like and HER2enriched breast cancers. While we could replicate their finding, we want to take this opportunity to clarify that the prognostication by ERK5 mRNA is in fact related to chemotherapy rather than node-positivity. We used all the probes which detect mRNA transcripts of ERK5 and found that the stratification of relapse-free survival (RFS) and distant-metastasis-free survival (DMFS) by ERK5 mRNA is relevant to both node-negative (N0) and node-positive (N1) ER-negative tumors only after chemotherapy (Table 1A). We also analyzed the association of ERK 5 mRNA with patient outcome in basal-like breast cancer. We found that ERK5 mRNA overexpression associates with poor RFS in patients who received chemotherapy, but not in patients who did not receive chemotherapy (Table 1B). Moreover, we found that considering the overexpression of MEK5 mRNA in addition to ERK5, as an indicator of the MEK5-ERK5 signaling axis, associated with poorer RFS and DMFS in basal-like breast cancer who received chemotherapy (Table 1B and Figure 1A). The combined MEK5-ERK5 mRNA expression also associated with DMFS of HER2-enriched patients who received systemic treatment but not those who were systemically untreated (Figure 1B). Our in silico analyses suggest that ERnegative and the basal-like and HER2-enriched intrinsic subtypes of breast cancer with low expression of MEK5- 
Table 1: ERK5 mRNA associates with outcome after chemotherapy

\begin{tabular}{|c|c|c|c|c|c|c|c|c|c|}
\hline \multirow{4}{*}{ A } & & \multirow{2}{*}{\multicolumn{4}{|c|}{ No chemo }} & \multirow{2}{*}{\multicolumn{4}{|c|}{ Chemo }} \\
\hline & & & & & & & & & \\
\hline & & \multicolumn{2}{|c|}{ No } & \multicolumn{2}{|c|}{ N1 } & \multicolumn{2}{|r|}{ No } & \multicolumn{2}{|c|}{ N1 } \\
\hline & & $H R$ & $p$ & $H R$ & $p$ & $H R$ & $p$ & $H R$ & $p$ \\
\hline \multirow{2}{*}{ RFS } & 35617_at & \multicolumn{2}{|c|}{0.85} & \multicolumn{2}{|c|}{0.16} & 9.593 & 0.0068 & 3.571 & 0.0043 \\
\hline & 207292_s_at & 0. & & \multicolumn{2}{|c|}{0.78} & 11.19 & 0.0035 & 2.537 & 0.0346 \\
\hline \multirow{2}{*}{ DMFS } & 35617_at & 0 . & & \multicolumn{2}{|c|}{0.27} & 9.900 & 0.0236 & 6.806 & \multirow[t]{2}{*}{0.0034} \\
\hline & 207292_s_at & 0 . & & & & 9.251 & 0.0272 & & \\
\hline \multirow[t]{3}{*}{ B } & & \multicolumn{5}{|c|}{ RFS } & \multicolumn{3}{|c|}{ DMFS } \\
\hline & & \multicolumn{2}{|c|}{ No chemo } & \multicolumn{3}{|c|}{ Chemo } & No chemo & \multicolumn{2}{|c|}{ Chemo } \\
\hline & & $H R$ & $p$ & & $H R$ & $p$ & $H R$ & $H R$ & $p$ \\
\hline \multirow{2}{*}{ ERK5 } & 35617_at & \multicolumn{2}{|c|}{0.93} & \multicolumn{2}{|c|}{4.0460} & 0.0027 & 0.46 & \multicolumn{2}{|c|}{0.06} \\
\hline & 207292_s_at & \multicolumn{2}{|c|}{0.44} & \multicolumn{2}{|c|}{3.3230} & 0.0012 & 0.97 & \multicolumn{2}{|c|}{0.06} \\
\hline & 204756_at & \multicolumn{2}{|c|}{0.65} & \multicolumn{3}{|c|}{0.14} & 0.71 & \multicolumn{2}{|c|}{0.48} \\
\hline MEK5 & 210482_x_at & & & & & & 0.63 & & \\
\hline & 211370_s_at & & & & & & 0.51 & & \\
\hline $\begin{array}{c}\text { MEK5- } \\
\text { ERK5 }\end{array}$ & Combined & & & & 970 & 0.0047 & 0.62 & 9.9860 & 0.0485 \\
\hline
\end{tabular}

A No Chemotherapy
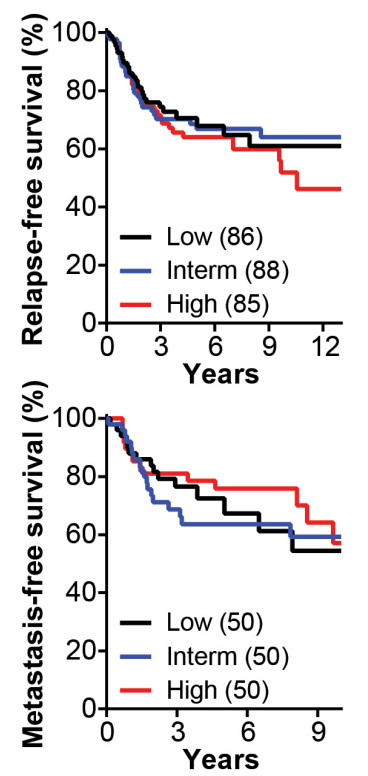

B No sys. treatment

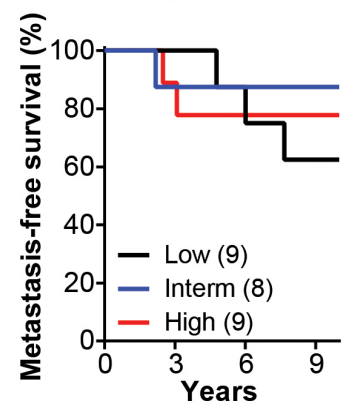

Chemotherapy

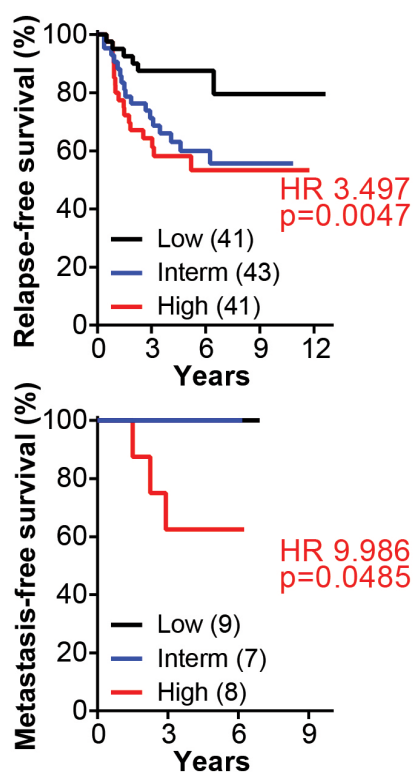

Sys. treatment

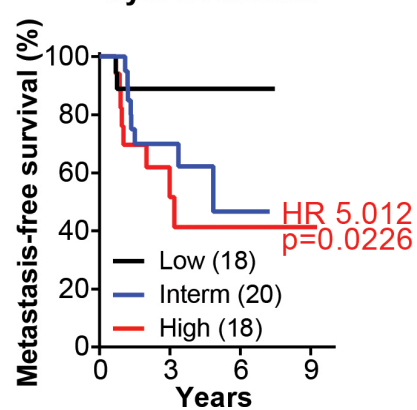

The KM Plotter Online Tool was used to investigate relapse-free survival (RFS) and distant metastasis-free survival (DMFS) in ERnegative (A) and basal-like (B) breast cancers. In A, patients were also separated according to nodal status (node-negative N0; node-positive N1). Hazard ratio (HR) and p-value (p) from log-rank (Mantel-Cox) test for each analysis were obtained using GraphPad® Prism by comparing patients with the highest expression (top 33\%; upper tertile) to those with the lowest expression (bottom 33\%; lower tertile). For the MEK5ERK5 analysis, the average of all the specified probes, which detect mRNA transcripts of MEK5 and ERK5, were used.

Figure 1: MEK5-ERK5 mRNA expression associates with poor survival after systemic treatments. (A) Relapse-free survival (top row) and distant metastasisfree survival (middle row) of basal-like breast cancer were analyzed in subsets of patients who did not receive or received chemotherapy (No chemotherapy vs. Chemotherapy). (B) Distant metastasis-free survival of HER2-enriched breast cancer in subsets of patients who did not receive systemic treatments or received systemic treatments (No sys. treatment vs. Sys. treatment). The KM Plotter Online Tool was used to carry out the analyses. Patients were stratified according to the average expression level of MEK 5 and ERK5. The number of patients in the lower tertile (low; bottom 33\%), the upper tertile (high, top $33 \%$ ) and the middle tertile (intermediate; interm) groups are indicated in parentheses. HR and log-rank p-value (p) for each analysis were obtained using $\mathrm{GraphPad}^{\circledR}$ Prism by comparing the survival of patients in the upper tertile (High) to those in the lower tertile (Low). 
ERK5 do benefit from systemic treatments including chemotherapy, whereas patients with high expression of MEK5-ERK5 do not. It is noteworthy that ERK5 protein expression has been previously associated with poorer survival in breast cancer [7].

In conclusion, we [3] and Ortiz-Ruiz et al. [4] found that ERK5 inhibition potentiates chemotherapy in vitro and in vivo. These findings along with the lack of benefit from chemotherapy in patients with MEK5-ERK5 overexpression support the rationale to inhibit MEK5ERK5 signaling pathway in combination with neoadjuvant and/or adjuvant chemotherapy in ER-negative, TNBC and basal-like breast cancer to improve survival rates.

\section{Conflict of interest}

The authors declare no conflict of interest.

\section{ACKNOWLEDGEMENT}

F.A. is supported by Future Fellowship from the Australian Research Council [ID: FT130101417], the Australian National Health and Medical Research Council [NHMRC, ID: APP1082458] and the Rio Tinto Ride to Conquer Cancer (RTCC)/Weekend to End Women's Cancers (WEWC) [ID: RTCC-WEWC15014].

\section{REFERENCES}

1. Yang Q, Lee JD. Targeting the BMK1 MAP kinase pathway in cancer therapy. Clin Cancer Res. 2011;17(11):3527-32.

2. Drew BA, Burow ME, Beckman BS. MEK5/ERK5 pathway: the first fifteen years. Biochimica et biophysica acta. 2012;1825(1):37-48.

3. Al-Ejeh F, Miranda M, Shi W, Simpson PT, Song $\mathrm{S}$, Vargas $\mathrm{AC}$, et al. Kinome profiling reveals breast cancer heterogeneity and identifies targeted therapeutic opportunities for triple negative breast cancer. Oncotarget. 2014;5(10):3145-58.

4. Ortiz-Ruiz MJ, Alvarez-Fernandez S, Parrott T, Zaknoen S, Burrows FJ, Ocana A, et al. Therapeutic potential of ERK5 targeting in triple negative breast cancer. Oncotarget. 2014;5(22):11308-18.

5. Goh KC, Novotny-Diermayr V, Hart S, Ong LC, Loh YK, Cheong A, et al. TG02, a novel oral multi-kinase inhibitor of CDKs, JAK2 and FLT3 with potent anti-leukemic properties. Leukemia. 2012;26(2):236-43.

6. Gyorffy B, Lanczky A, Eklund AC, Denkert C, Budczies J, $\mathrm{Li} \mathrm{Q}$, et al. An online survival analysis tool to rapidly assess the effect of 22,277 genes on breast cancer prognosis using microarray data of 1,809 patients. Breast cancer research and treatment. 2010;123(3):725-31.

7. Montero JC, Ocana A, Abad M, Ortiz-Ruiz MJ, Pandiella A, Esparis-Ogando A. Expression of Erk5 in early stage breast cancer and association with disease free survival identifies this kinase as a potential therapeutic target. PLoS ONE. 2009;4(5):e5565. 